\title{
Rice leaf inclination2, a VIN3-like protein, regulates leaf angle through modulating cell division of the collar
}

\author{
Shu-Qing Zhao ${ }^{1, *}$, Jiang $\mathrm{Hu}^{2, *}$, Long-Biao Guo ${ }^{2}$, Qian Qian ${ }^{2}$, Hong-Wei Xue ${ }^{1}$

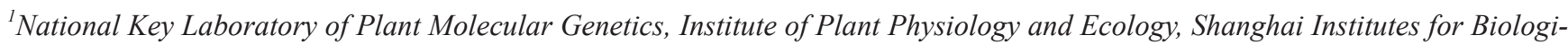 \\ cal Sciences, Chinese Academy of Sciences, Shanghai 200032, China; ${ }^{2}$ State Key Laboratory of Rice Biology, China National Rice \\ Research Institute, Hangzhou 310006, Zhejiang, China
}

As an important agronomic trait, inclination of leaves is crucial for crop architecture and grain yields. To understand the molecular mechanism controlling rice leaf angles, one rice leaf inclination2 (lc2, three alleles) mutant was identified and functionally characterized. Compared to wild-type plants, $l c 2$ mutants have enlarged leaf angles due to increased cell division in the adaxial epidermis of lamina joint. The $L C 2$ gene was isolated through positional cloning, and encodes a vernalization insensitive 3-like protein. Complementary expression of $L C 2$ reversed the enlarged leaf angles of $l c 2$ plants, confirming its role in controlling leaf inclination. $L C 2$ is mainly expressed in the lamina joint during leaf development, and particularly, is induced by the phytohormones abscisic acid, gibberellic acid, auxin, and brassinosteroids. LC2 is localized in the nucleus and defects of LC2 result in altered expression of cell division and hormone-responsive genes, indicating an important role of LC2 in regulating leaf inclination and mediating hormone effects.

Keywords: leaf inclination; rice; VIN3-like protein; cell division; LC2

Cell Research (2010) 20:935-947. doi:10.1038/cr.2010.109; published online 20 July 2010

\section{Introduction}

Leaf inclination, the angle between the leaf blade and the culm, is an important agronomic trait in monocotyledonous plants, and contributes to the plant architecture and grain yields $[1,2]$. It has been shown that an erectleaf trait in rice enhances the efficiency of sunlight capture and increases the nitrogen reservoirs for grain filling, and renders the plants more suitable for dense plantings $[2,3]$.

During rice growth, after the complete elongation of the leaf blade and leaf sheath, the region between them, designed as the lamina joint or collar, begins to develop, resulting in the leaf blade bending away from the vertical and the formation of the leaf angle [1]. The lamina joint contributes significantly to the blade bending horizon-

\footnotetext{
*These two authors contributed equally to this work.

Correspondence: Hong-Wei Xue ${ }^{\mathrm{a}}$, Qian Qian ${ }^{\mathrm{b}}$

a'E-mail: hwxue@sibs.ac.cn

bE-mail: qianqian188@hotmail.com

Received 13 January 2010; revised 19 March 2010; accepted 19 March 2010; published online 20 July 2010
}

tally from the main axis, and abnormal development of the collar will result in changed leaf angles. Lack of longitudinal elongation in the collar resulted in the erect leaf [4]; conversely, increased cell expansion of collar adaxial cells resulted in the enhanced leaf inclination $[5,6]$, indicating the importance of collar development in leaf angle formation.

To date, several rice mutants with altered leaf inclination or QTLs related to lamina joint angle have been genetically identified [3, 7-10], and the major detected QTL for leaf angle is tiller angle 1 (tal) [7, 8]. However, the underlying mechanism of the leaf angle alteration has not been clarified. Most of the identified mutants with altered leaf angles are related to the biosynthesis or signaling of the phytohormone brassinosteroids (BRs). Rice lamina joint angle increases proportionally to BR levels $[11,12]$, and deficiency of OsDWARF4, a key gene in BR biosynthesis, resulted in increased leaf erectness and enhanced grain yields in dense planting [3]. The erect leaves were also observed in other BR biosynthesis-defective mutants including ebisu $d$ warf ( $d 2$, deficiency of CYD90D2/D2 [10]) and brassinosteroid-deficient dwarf1 (brdl, deficiency of OsDWARF [9]). In addition, the BR 
signaling-defective mutant $d 61-7$, the weakest mutant of rice BRASSINOSTEROID INSENSITIVE1 (OsBRI1, a protein kinase severing as rice BR receptor), and transgenic rice plants with suppressed expression of $O s B Z R 1$ (a transcription factor involved in BR signaling pathway), showed erect leaves [13-15]. Conversely, transgenic rice plants overexpressing sterol C-22 hydroxylase, which catalyzes a rate-limiting step in BR biosynthesis, showed a slightly sprawling phenotype with increased leaf angles $[16,17]$. These results indicate the critical effects of BR, both biosynthesis and signaling, in regulating leaf inclination.

Besides BR, many other phytohormones are involved in controlling the lamina joint inclination. Ethylene participates in the response of BR-induced rice lamina inclination, and auxin (IAA) affects the lamina joint inclination at high concentrations and has a synergistic interaction with BR $[5,11]$. In addition, suppression of the expression of SPINDLY (a negative regulator of gibberellin signaling) by antisense and RNAi approaches led to increased lamina joint bending [18].

Many transcription factors affect the rice leaf inclination. Ectopic expression of LAX PANICLE (LAX), a basic helix-loop-helix transcription factor, increased the rice lamina joint bending [19]. Overexpression of BRASSINOSTEROID UPREGULATED 1 (BU1), a helix-loop-helix transcription factor involved in BR signaling in rice, results in the enhanced lamina joint bending whereas the RNAi plants with suppressed $B U 1$ expression showed the erected leaf phenotype [20]. OSLIGULELESS1 (OSLG1) encodes a transcription factor containing an SQUAMOSA promoter binding protein domain, and its deficiency mutant, oslg1, shows defect in the ligule, auricle, and laminar joint [21]. Analysis of the leaf angle mutant large leaf angles (lla), a T-DNA insertion mutant, showed that OsWRKY11, a WRKY transcription factor, also regulates leaf inclination [22]. Rice short vegetative phase group MADS-box proteins, OsMADS22, OsMADS55 and OsMADS47, negatively regulate leaf joint angles and are negative regulators of BR responses $[6,23]$. Downregulation of $O s L I C$, which encodes a $\mathrm{CCCH}$-type zinc-finger protein with transcription activation activity, results in the increased angles of rice leaf and tiller through regulating the BR signaling [24]. The pair of antagonistic HLH/bHLH transcription factors, ILI1 and IBH1, mediates the BR regulation of lamina inclination in rice [25]. In addition, increased expression of the OsAGO7 gene, the ortholog of the Arabidopsis ZIPPY (ZIP/AGO7, belonging to the Argonaute family), resulted in the upward curling of the leaf blade and enhanced erectness of the leaf [26].

Regulation of development and inclination of leaves involves multiple factors and complex regulatory networks. To further elucidate the relevant mechanism, we identified a rice mutant leaf inclination 2 ( $l c 2$, three alleles), which had increased leaf angles due to increased cell division in collar adaxial epidermis. Isolation of $L C 2$ through map-based cloning and functional studies revealed that $\mathrm{LC} 2$, a vernalization insensitive 3 (VIN3)like protein, functions as a repressor of cell division to regulate the collar development and hence the leaf angle of rice.

\section{Results}

Identification of rice lc2 mutant, which has enlarged leaf inclination due to enhanced adaxial cell division at the lamina joint

To identify key factors regulating rice leaf inclination, a mutant $l c 2$ (three alleles $l c 2-1,-2$, and -3 ), which has an obviously exaggerated leaf angle, was identified as a result of naturally occurring mutation. Phenotypic observation showed that after the complete development of the third leaf, leaf angles of $l c 2$ were much larger compared to the wild-type (WT) (Figure 1A, left panel). This was more evident at the heading stage (reaching to $\sim 42{ }^{\circ}$ in $l c 2-1$, comparing to $19^{\circ}$ in WT, Figure 1A, right panel; $1 \mathrm{C})$.

It has been shown that the lamina joint contributes significantly to the leaf angle formation and enlarged leaf angles may reflect alterations of lamina joint development $[5,13]$. Indeed, observation of lamina joint morphology (Figure 1D, left panel), measurement of the length of abaxial and adaxial epidermis, and calculation of the adaxial/abaxial length ratio of $l c 2-1$ (1.98) and WT (1.32) (Figure 1D, right panel) confirmed that the main difference was due to the much-elongated adaxial surface of $l c 2-1$ ( $\sim 32 \%$ longer). Further morphological observation, through scanning electron microscopy, showed that, compared to the smooth adaxial surface of the lamina joint in the WT, that in $l c 2-1$ was much larger and formed a bulge, indicating an altered development of the lamina joint in $l c 2$ (Figure 1E).

Previous studies showed that abnormal cellular development, such as lacking the longitudinal elongation or increased cell expansion of the adaxial cells in the collar, will result in altered leaf angles [4-6]. Indeed, observation of the cross and longitudinal sections revealed increased cell layers at the adaxial surface of the $l c 2-1$ lamina joint, indicating that the enlarged leaf inclination resulted from increased cell division (Figure 1E). In addition, the cell length in the longitudinal sections of the lc2-1 collar was unaltered (Figure 1F), confirming that the enlarged leaf angle of $l c 2-1$ was due to increased cell 


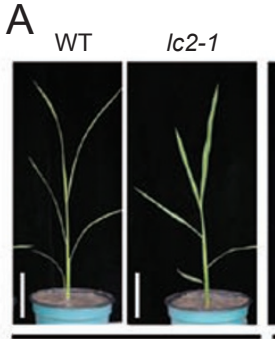

3 weeks

B

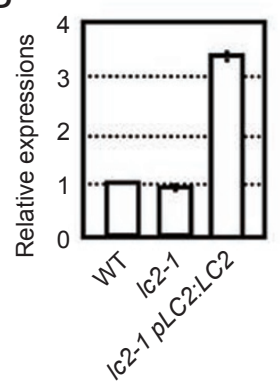

$\mathrm{D}$

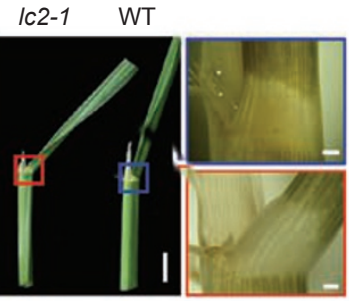

E

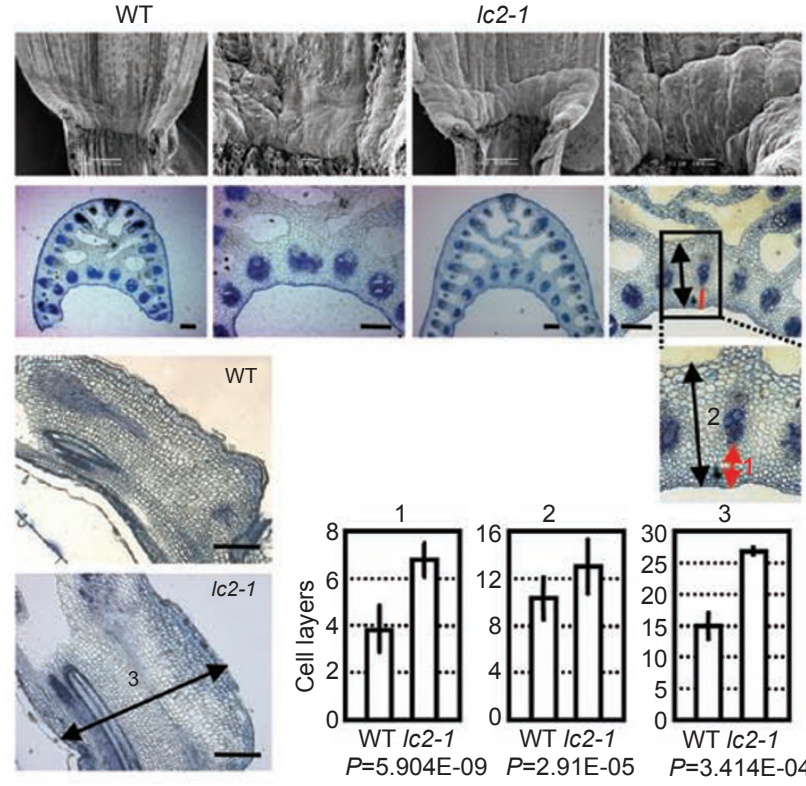

C

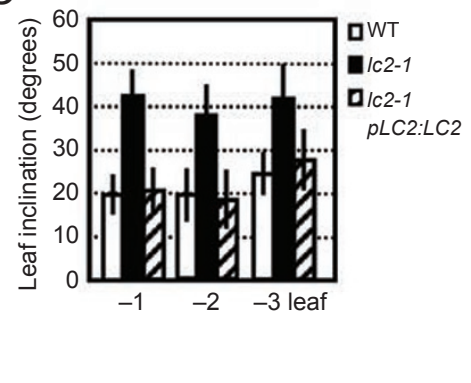

$\mathrm{F}$
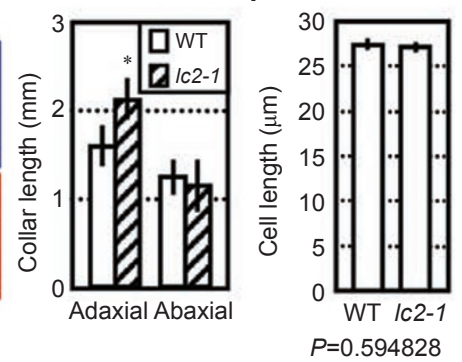

Figure 1 Phenotypes of IC2. (A) Growth of the wild-type (YunDao32, same in other figures) and the Ic2-1 plants at 3 weeks (left panel, bar $=5 \mathrm{~cm}$ ), and growth of WT, Ic2-1 plants and Ic2-1 plants with complemented expression of $L C 2$ at heading stage (right panel, bar $=10 \mathrm{~cm}$ ). (B) qRT-PCR analysis on the $L C 2$ transcripts in the lamina joint of WT plants, Ic2-1 mutants and Ic2-1 plants with complementary expression of $L C 2$. $L C 2$ transcript levels were normalized to that of $A C T I N$ and relative expressions were compared with that of WT plants. Means values were obtained from three independent PCR amplifications. Error bars indicate SE. (C) Leaf inclination of last three leaves at the heading stage of WT plants, Ic2-1 mutants and Ic2-1 plants with complementary expression of $L C 2$. The leaf angles were measured and presented as means \pm SE $(n>20)$. (D) Comparison of the leaf inclination of WT and Ic2-1 mutant at the heading stage (left panel, bar $=1 \mathrm{~cm}$ ). The differences at lamina joints were highlighted (squares, middle, bar $=200$ $\mu \mathrm{m})$. Collar lengths of adaxial and abaxial surfaces of the flag leaf were measured and ratio (adaxial/abaxial) was calculated. The data were presented as means \pm SE $(n>9)$ and statistically calculated by Student's $t$-test $(* P<0.05)$. (E) Morphology of adaxial surface (upper panel), cross sections (middle panel), and longitudinal sections (lower panel, left) through the lamina joint of flag leaves of WT and Ic2-1 plants. The adaxial cell layers of the cross sections (indicated as 1 and 2) and cell layers of the longitudinal sections (indicated as 3 ) through the lamina joint were measured and statistically calculated by Student's $t$ test. Error bar represents SE $(n>100)$. Bar $=200 \mu \mathrm{m}$. (F) Adaxial cell length of longitudinal sections (as indicated in E) through the lamina joint of flag leaves of WT and Ic2-1 plants. Error bar represents SE, and Student's $t$-test revealed the unaltered cell elongation.

has other alterations during plant development, including dark color and reversely rolled leaves, late flowering, and severe sterility. Calculation of the heading time by the formation of young panicles showed that flowering time was significantly delayed in the $l c 2-1$ mutant in comparison with that of WT (Figure 2A). Compared with WT plants, the leaf blades of $l c 2$ mutant began to roll reversely after growth of the third leaf and became more obvious as leaf development progressed (Figure 2B). Examination of the leaf histology through cross sections of flag leaves at the heading stage showed that compared with that in WT plants, the number of bulliform cells was increased in $l c 2$ mutant (Figure 2B), which may contribute to the rolling of the leaf blade.

\section{Map-based cloning of LC2}

Genetic assays with reciprocal crosses between $l c 2$ and WT plants showed that $l c 2$ has a recessive mutation in a single locus. Allelic tests between the $l c 2$ and other mutants indicated that $L C 2$ is a previously unknown locus involved in rice leaf inclination regulation. Furthermore, the $L C 2$ gene was identified by map-based

division

Besides the increased leaf inclination, the $l c 2$ mutant 
A

WT
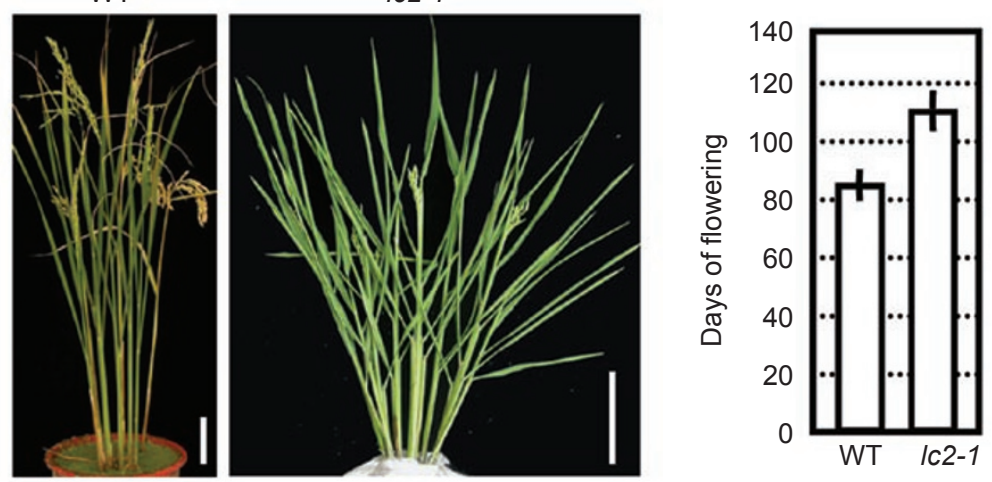

B
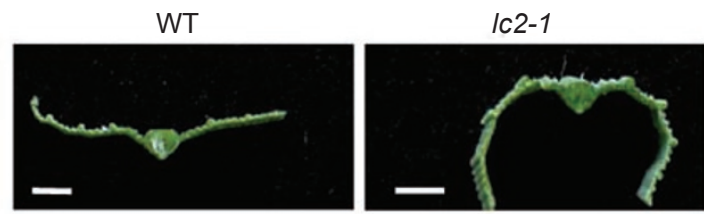

IC2-1 pLC2:LC2
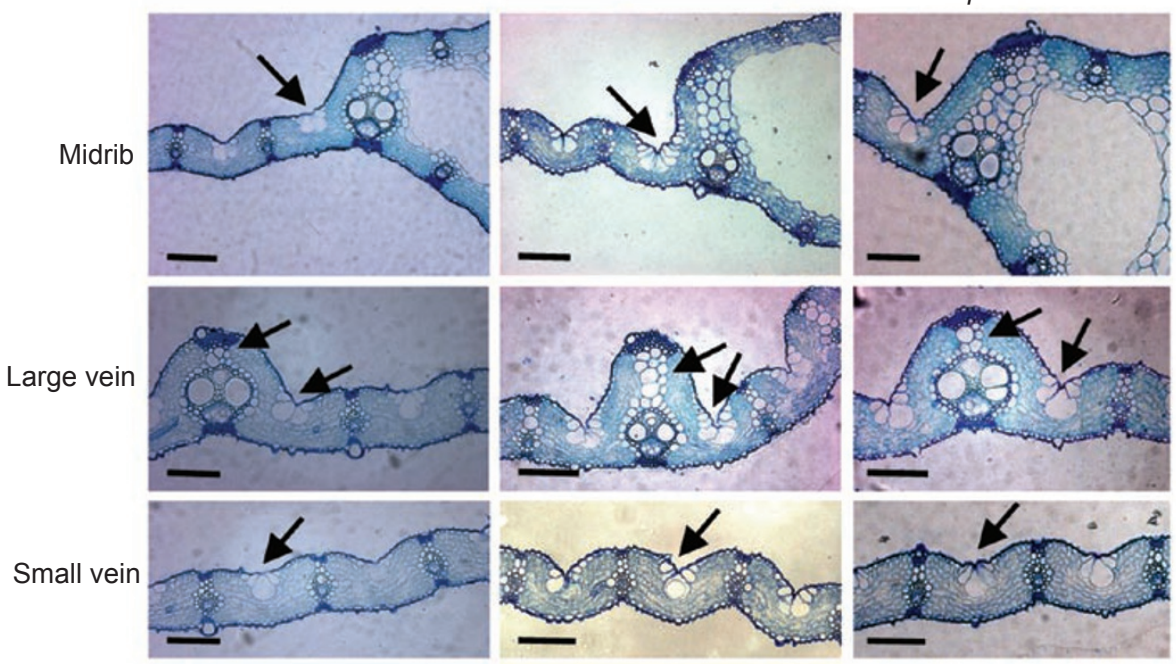

Figure 2 Ic2 has reversely rolled leaves and excessive bulliform cells, and delayed flowering. (A) Observation (left panel, bar $=10 \mathrm{~cm}$ ) and calculation (right panel) analysis show that $/ c 2-1$ mutant plants have delayed flowering when compared with WT plants. The WT (YunDao32) and Ic2-1 plants were grown in a phytotron with a $12 \mathrm{~h}$ light $\left(28^{\circ} \mathrm{C}\right) / 12 \mathrm{~h}$ dark $\left(22^{\circ} \mathrm{C}\right) \mathrm{cycle}$. The flowering time is presented as means \pm SE $(n>10)$ and the experiments are biologically repeated. (B) Ic2-1 mutant plants have slightly reversely rolled leaves (upper panel, bar $=500 \mu \mathrm{m}$ ), and cross sections of the flag leaf blade of WT and $/ c 2-1$ plants at heading stage revealed the increased numbers of bulliform cells around the midrib, large vein, and small vein (lower panel, bar $=100 \mu \mathrm{m})$. Complemented expression of $L C 2$ recovers the altered numbers of bulliform cells.

cloning in an F2 population by crossing $1 c 2-1$ (japonica) and MingHui63 (indica). The $L C 2$ gene was initially located on the distal end of the short arm of chromosome 2 between simple sequence repeat (SSR) marker RM7451 and sequence-tagged site (STS) marker HS15. It was then mapped on two overlapping bacterial artificial chromosome (BAC) clones, OSJNBa0050G13 and P0463E12, in an interval of $64 \mathrm{~kb}$ between the STS markers HS37 and HS52 and co-segregated with the marker HS45, by using $1741 \mathrm{~F} 2$ homozygous recessive plants (Figure $3 \mathrm{~A})$. To define the mutated locus, corresponding DNA fragments of the WT $L C 2$ gene and the $l c 2-1, l c 2-2$ and $l c 2-3$ alleles were amplified and sequenced. Comparison of the allelic gene sequences revealed that the $1 c 2-1$ allele contains a 2-bp deletion at positions +1558 to 1559 bp (CA) of the LOC_Os02g05840 open reading frame (ORF), which is in the fourth exon and causes the shift of the reading frame; the $l c 2-2$ allele carries a segment 
substitution at position +2044 to $2050 \mathrm{bp}$ (CAGGAAGA to TAAGTTTCTGACT); and the $l c 2-3$ allele has a 29 -bp deletion at position +2 122 to $2150 \mathrm{bp}$ (TAC GTA AAC ACT CTT ATT GAT GAT CCT GT, Figure 3B). All these three alleles result in premature translational stops.

LC2 encodes a VIN3-like protein, which could functionally rescue the lc2 phenotype

Comparison of the genomic DNA and correspond- ing cDNA sequences revealed that $L C 2$ consists of four exons and encodes a 749 amino-acid protein (the corresponding full-length cDNA was identified as AK101341 in Knowledge-based Oryza Molecular biological Encyclopedia). Analysis through Basic Local Alignment Search Tool indicated that the LC2 protein shares the highest identity ( $74 \%$ over the entire protein) with TmVIL2, a predicted member of the VIN3-like protein in wheat, and 37\% identity with AtVIL2 and AtVIN3 [27-29].

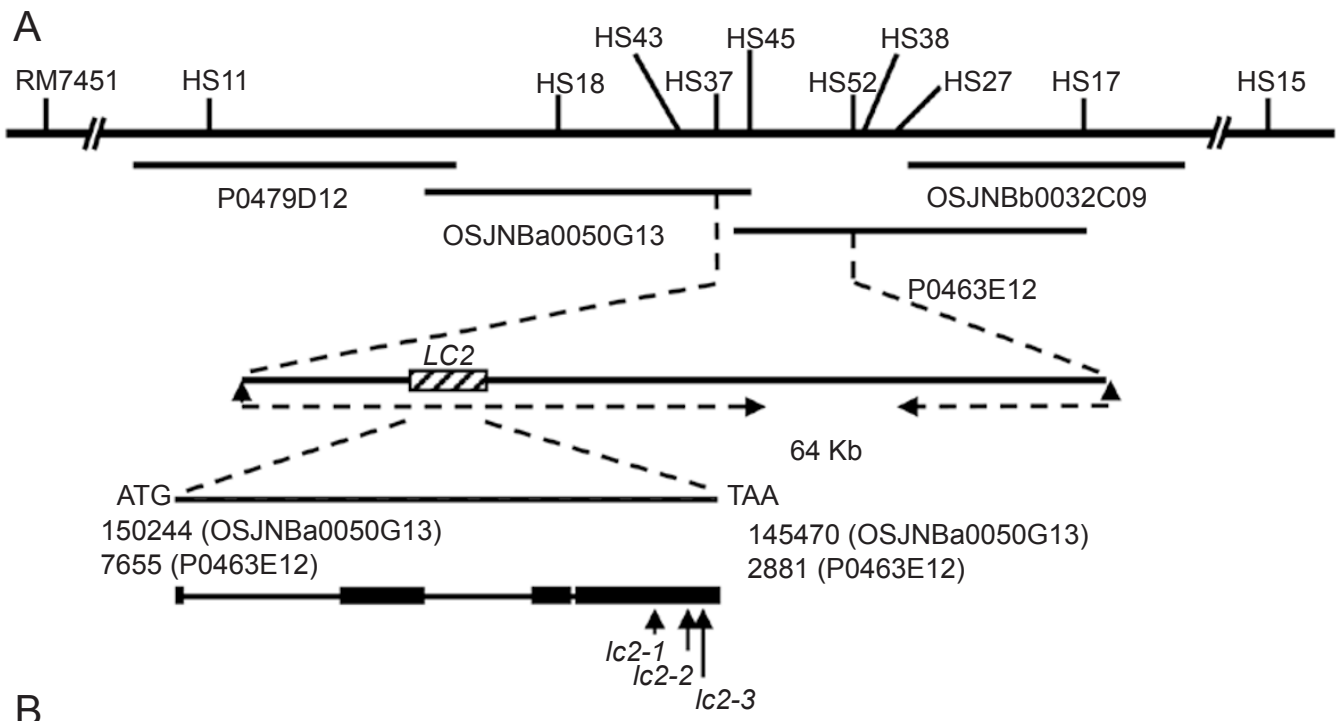

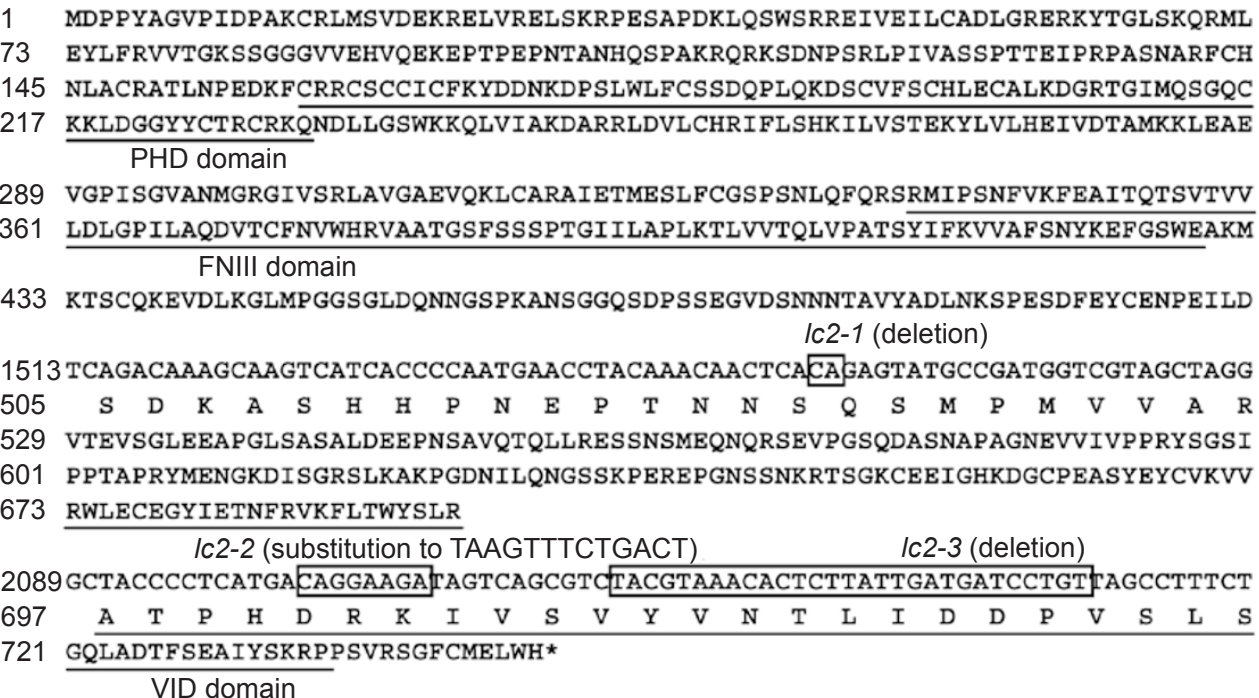

Figure 3 Map-based cloning and conformation of the LC2 functions. (A) The LC2 locus was primarily mapped in the short arm of rice chromosome 2 between markers RM7451 and HS15. A BAC contig covering the LC2 locus and the numeral indicate the numbers of recombinants identified from 1741 lc2-1 F2 plants. LC2 locus was narrowed to a 64 kb genomic DNA region (overlap of BACs OSJNBa0050G13 and P0463E12). The start codon (ATG) and stop codon (TAA) are indicated. Closed boxes and lines indicate the exons and introns, respectively. The mutated sites in Ic2-1, Ic2-2, and Ic2-3 are shown. (B) Predicted amino-acid sequences of LC2. There is a 2-bp deletion in Ic2-1 and 29-bp deletion in Ic2-3, and a segment substitution in Ic2-2. Sequences of the predicted PHD, FNIII, and VID domains are underlined. 
Previous structural analysis has shown that VIN3like proteins contain several highly conserved elements including a plant homeodomain finger motif (PHD, characterized by a conserved Cys4-His-Cys3 pattern and known to be associated with chromatin-mediated transcriptional regulation $[27,30,31])$, a fibronectin type III domain (FNIII, one of the repeated structural motifs discovered in fibronectin and involved in protein-protein interactions [32, 33]), and a VIN3-interacting domain (VID, conserved among all members of this family [29]). Indeed, structural analysis revealed that LC2 contains all these conserved domains (Figure 4A), and the premature stops caused by the $l c 2$ mutations result in full $(l c 2-1)$ or partial $(l c 2-2,3)$ deletion of the VID domain (Figure 3B). Analysis of the polygenetic relationships showed that the LC2 protein was closer to TmVIL2 and OsVIL4 (Figure 4B).

To confirm the enlarged leaf inclination in the mutant was due to deficiency in $L C 2$, complementation analysis was performed by transforming a binary vector carrying a 7.4-kb WT genomic fragment containing the entire LC2 ORF along with a 1.8-kb upstream promoter region, to the $l c 2-1$ mutant. Phenotypic observation of the transgenic $l c 2-1$ plants (65 independent transgenic lines were obtained, Figure 1B) indicated that the normal leaf inclination was restored and there were normal numbers of bulliform cells (Figure 1A, right panel; 1C, Figure 2 ), thus showing that $L C 2$ indeed functions in leaf angle regulation and leaf rolling control.

LC2 is expressed in aerial parts and induced by hormones

Quantitative real-time RT-PCR (qRT-PCR) and promoter-reporter gene fusion analyses were performed to study the expression pattern of $L C 2$. qRT-PCR analysis revealed that $L C 2$ was expressed in various tissues, with a high level of expression in leaf, medium expression in seedling, root and old flowers, and low expression in stem and young flowers (Figure 5A, left panel). Furthermore, a $1.8 \mathrm{-kb}$ promoter region of $L C 2$, which was also used in the complementation studies, was fused to the $\beta$-glucuronidase $(G U S)$ gene and used for rice transformation. Eleven independent transgenic lines were identified and histochemical analysis of GUS activities revealed that the $L C 2$ promoter drives gene expression in the overground parts of the seedling (coleoptile during seed germination), lamina joints and anthers (Figure 5A, right panel). In the mature leaf, $L C 2$ was specifically expressed in the joint region between leaf blade and sheath, consistent with its role in lamina joint development.

Due to its critical roles in lamina joint development, expression of $L C 2$ in collar was further analyzed through
qRT-PCR analysis. The results showed that $L C 2$ was expressed in all the leaf collars and was relatively highly transcribed in the early appearing leaves (1st-5th leaves, compared to 6-11th leaves, Figure 5B, upper panel). In addition, analysis of $L C 2$ expression in the last four (8-11th) leaves at different developmental stages revealed that, although the expression levels varied in different collars, there was a tendency: $L C 2$ was expressed
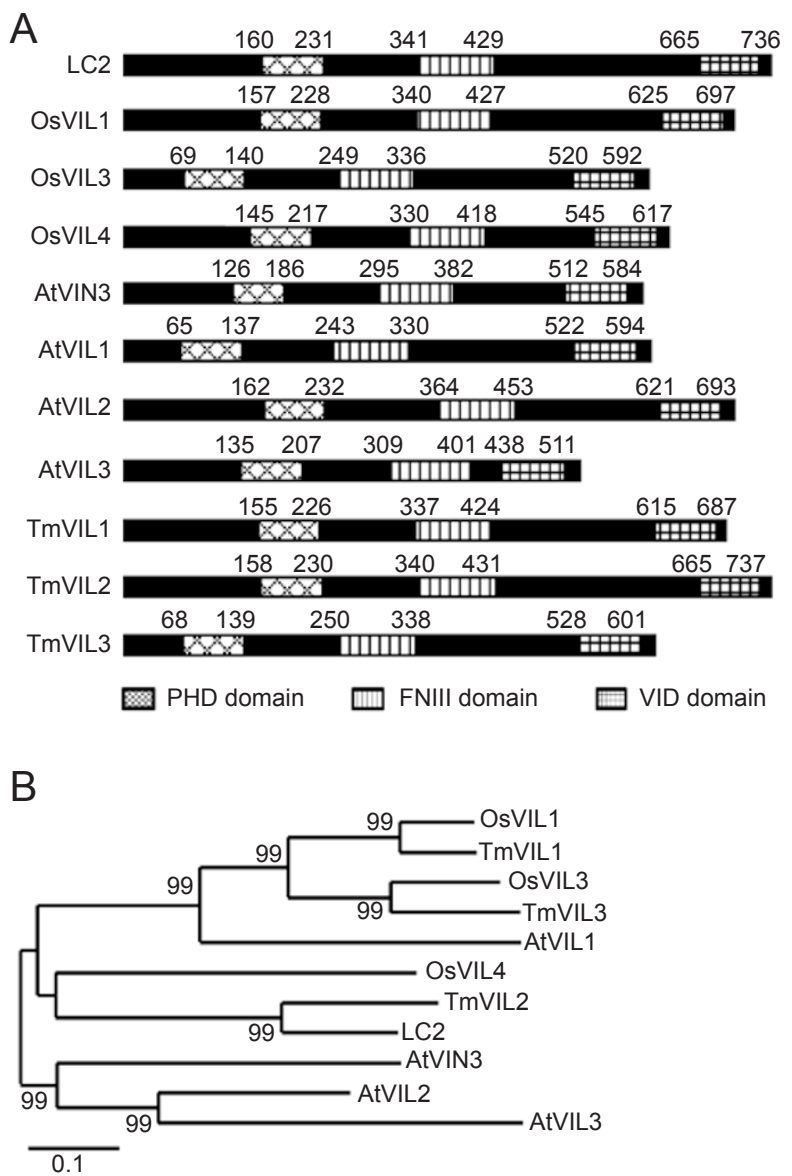

Figure 4 Structural organization and phylogenetic relationship of the VIN3/VIL proteins. (A) Structural organization of the VIN3/VIL proteins. VIN3/VIL proteins from Arabidopsis, rice, and wheat were analyzed. Conserved domains including PHD, FNIII, and VID domains are indicated. Accession numbers are as follows: LC2 (Oryza sativa, BAD38062), OsVIL1 (O. sativa, ABA98812) OsVIL3 (O. sativa, AAT94000), OsVIL4 (O. sativa, BAD03519), TmVIL1 (Triticum monococcum, DQ886919), TmVIL2 (T. monococcum, DQ886917), TmVIL3 (T. monococcum, DQ886918), AtVIN3 (Arabidopsis thaliana, AAR91717), AtVIL1 (A. thaliana, NP_189087), AtVIL2 (A. thaliana, BAB17836) and AtVIL3 (A. thaliana, AAX23819). The numbers indicate the amino-acid positions of the corresponding domains. (B) Phylogenetic tree of the VIN3/VIL proteins. The tree was generated using Clastal. 

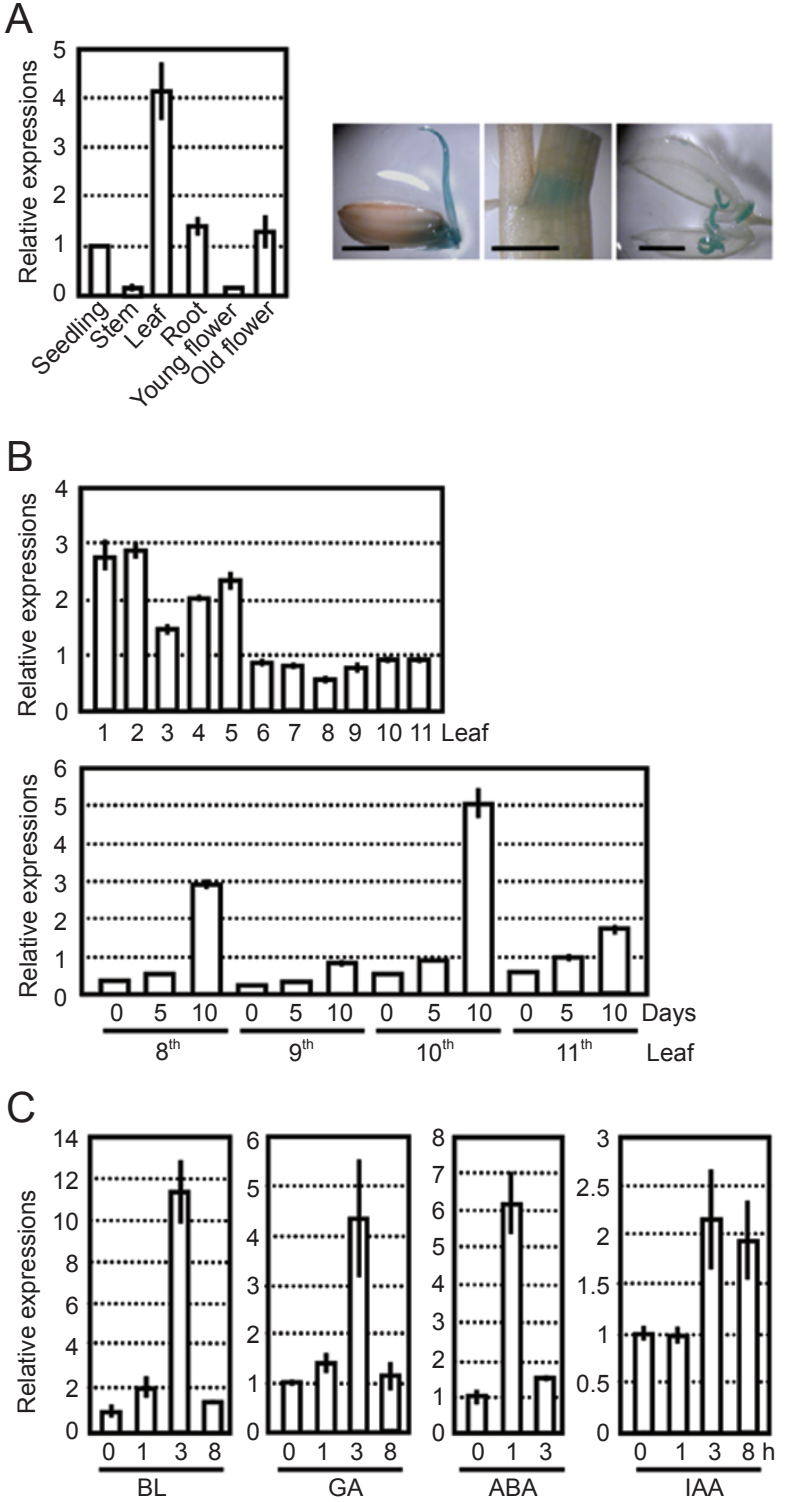

at a low level in early stages, and its expression increased along with collar development and leaf maturation (Figure $5 \mathrm{~B}$, lower panel).

In addition, $L C 2$ expression is induced by multiple hormones. Analysis of the transcript levels showed that $L C 2$ was rapidly induced by 24 -epibrassinolide (24-eBL) and $\mathrm{GA}_{3}$ ( 1 and $3 \mathrm{~h}$, recovered after $8 \mathrm{~h}$ ), relatively stably induced by IAA (after $3 \mathrm{~h}$ ), and transiently induced by abscisic acid (ABA; recovered after $3 \mathrm{~h}$, Figure 5C).

\section{Increased expression of cell-division-related genes under LC2 deficiency}

To further investigate the functional mechanism of $L C 2$ regulation of cell division at the lamina joint, expressions of genes known to be involved in cell division
Figure 5 Expression patterns of $L C 2$. (A) Left panel: qRT-PCR analysis of $L C 2$ transcripts in various tissues, including 2-weekold seedling, stems, leaf (fourth leaf), root of 2-week-old seedling, young flower (with a panicle size of 1-5 cm) and old flower (flower collected at full heading time). LC2 transcript levels were normalized to that of ACTIN and relative expressions were compared with that of 2-week-old seedling. Mean values were obtained from three independent PCR amplifications. Error bars indicate SE. Right panel: promoter-reporter gene (GUS) fusion studies further revealed the expressions of $L C 2$ in seedling, lamina joint, and flower (bar $=2.5 \mathrm{~mm}$ ). (B) qRT-PCR analysis on LC2 transcripts in collars of different leaves (from the first complete leaf, the collars were collected at 5 days after the complete elongation of leaf blade and leaf sheath, top panel), or last four leaves at different developmental stages (collars were collected at 0,5 , and 10 days after the complete elongation of leaf blade and leaf sheath of 8-11 leaves, bottom panel). Transcript levels of $L C 2$ gene were normalized with that of ACTIN. Mean values were obtained from three independent experiments. Error bars indicate SE. (C) qRT-PCR analysis on LC2 expression in seedlings treated with exogenous 24-epibrassinolide (BL, $1 \mu \mathrm{M}), \mathrm{GA}_{3}$ $(10 \mu \mathrm{M}) \mathrm{ABA}(100 \mu \mathrm{M})$, or IAA $(10 \mu \mathrm{M})$ for various times. $L C 2$ transcript levels were normalized to that of $A C T I N$ and relative expressions were compared with that of $0 \mathrm{~h}$. Mean values were obtained from three independent PCR amplifications. Error bars indicate SE.

or elongation in rice were studied. As shown in Figure $6 \mathrm{~A}, R 2$ (encoding a cyclin-dependent kinase-activating kinase, which regulates S-phase progression and controls cell division [34]) expression was three times higher in lc2-1 plants compared to WT, which was restored to normal level by complementary expression of $L C 2$. Analysis of the expression of $C D K A ; 1$ and $C D K A ; 2$ (two known $c d c 2$ protein kinases; $C D K A ; 2$ is possibly responsible for the induction of $\mathrm{G}_{1}$ - to $\mathrm{S}$-phase transition $[35,36]$ ) revealed that the pattern of $C D K A ; 2$ was similar to $R 2$ (Figure 6A, upper panel), whereas the transcript level of $C D K A ; 1$ was not obviously altered (Figure 6A, bottom panel). However, $H 4, H 3$ (two molecular markers for the S phase [36]), and EXPB6 (a member of the $\beta$-expansin gene family critical for cell elongation in deep water rice [37]) expressions were not obviously altered in the $l c 2-1$ mutant (Figure 6A, bottom panel).

Analysis of the expression of cell-elongation-related genes including XTR1 (encoding xyloglucan endotransglycosylase, the cell-wall loosening enzyme necessary for cell elongation [38]) showed that XTRI expression was enhanced in the mutant (Figure 6A, upper panel). Collectively, the results of our study suggest that $L C 2$ mainly regulates cell division through suppressing the expression of cell-division-related genes.

Interestingly, the expression of $L C 2$ is induced by $\mathrm{BR}$, $\mathrm{GA}$, and IAA. Examination of the transcripts of BR re- 


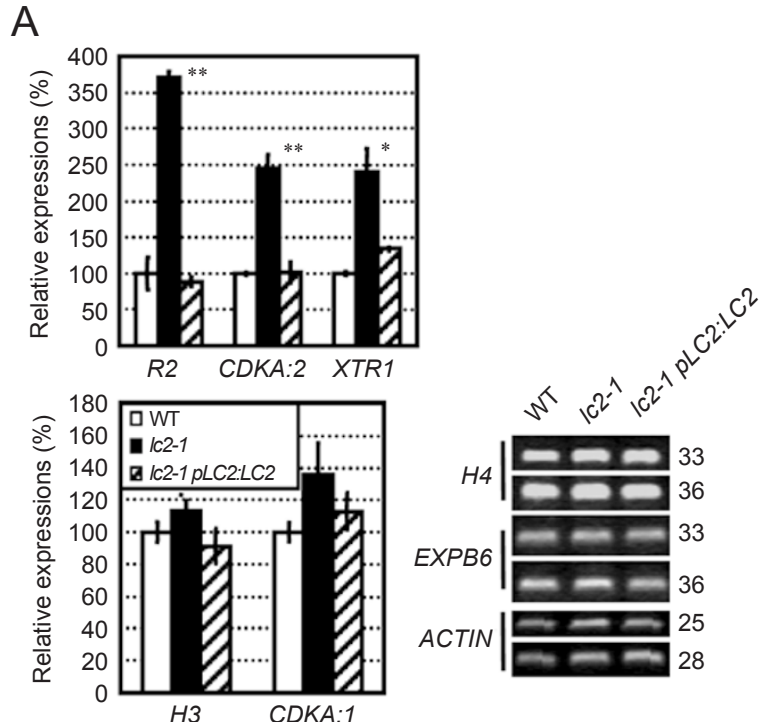

B

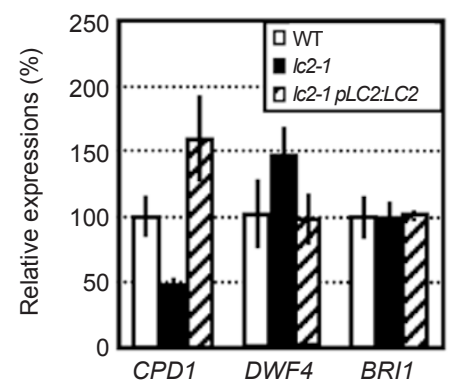

C

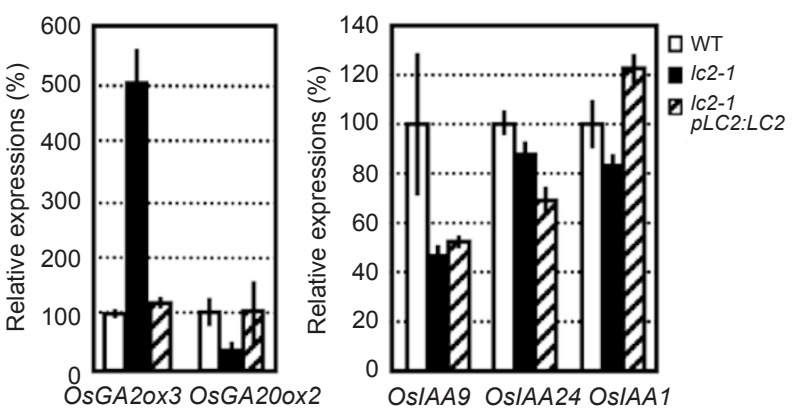

ceptor-encoding gene $B R I 1$ and BR-biosynthesis-related genes CPD1 and DWARF4 revealed little changes except that $C P D 1$ expression is reduced in $l c 2-1$ mutant (Figure 6B), suggesting a possibly enhanced BR signaling. In addition, analysis of the expression of GA metabolismrelated genes $O s G A 20 x 3$ and $O s G A 200 x 2$ revealed increased GA biosynthesis in $l c 2-1$ (Figure 6C, left panel) [39] and hence a possibly suppressed GA signaling. Although $L C 2$ is also induced by IAA, examination of the expression of IAA signaling-related genes OSIAAI, OsIAA9, and OsIAA24 revealed no obvious changes in lc2-1 (Figure 6C, right panel [40]), suggesting that LC2
Figure 6 Altered expressions of genes related to cell division or elongation in Ic2-1. The rice ACTIN gene was used as an internal positive control and transcript levels of the tested genes were normalized with that of ACTIN. Relative expressions of the tested genes were compared with that in WT plants. Means values were obtained from three independent experiments, and were statistically analyzed $\left({ }^{*} P<0.01 ;{ }^{*} P<0.005\right)$. Error bars indicate SE. (A) qRT-PCR and semiquantitative RT-PCR analyses were performed to study the transcript levels of cell division or elongation related genes in the lamina joints of flag leaves of WT, Ic2-1, and Ic2-1 pLC2:LC2 plants at the heading stage. The tested genes include $R 2, C D K A ; 2$ and $X T R 1$ (upper panel), and $H 3$ and $C D K A ; 1$ (bottom panel), and $H 4$ and EXB6 genes (by semiquantitative RT-PCR, bottom panel). (B) qRT-PCR analysis of the transcripts of BR-related genes in the lamina joints of WT, Ic2-1, and Ic2-1 pLC2:LC2 plants at heading stage. (C) qRTPCR analysis of the transcripts of GA- and IAA-related genes in the lamina joints of WT, Ic2-1, and Ic2-1 pLC2:LC2 plants at heading stage.

may not be involved in auxin signaling.

\section{LC2 is localized in nucleus}

The PHD finger is a common structural motif found in all eukaryotic genomes and proteins containing PHD finger are found universally in the nucleus [41]. The subcellular localization of LC2 was then determined through observing the transgenic rice plants or onion epidermal cells transiently transformed through particle bombardment, expressing the LC2-green fluorescent protein (GFP) fusion protein. Results showed that while GFP alone exhibited uniformly distributed fluorescence, LC2-GFP showed nuclear fluorescence only (Figure 7), indicating that LC2 is accumulated in the nucleus.

\section{Discussion}

Previous studies of VIN3-like proteins focused on their roles in vernalization of Arabidopsis and wheat [28, 29]. Arabidopsis VIN3 is a component of the polycomblike protein complex and is responsible for vernalizationmediated repression of the FLC gene, and vin3 mutant shows the late-flowering phenotype only after vernalization [42]. VIN3-LIKE 1 (VIL1) participates in both the photoperiod and vernalization pathways [28], suggesting the critical role of VIN3-like proteins in regulation of flowering [28, 42, 43]). In this study, we showed that spontaneous mutants of $L C 2$, a rice homolog of Arabidopsis VIN3, present the late-flowering phenotype, suggesting that even in rice, a nonvernalized plant, the VIN3-like protein also participates in flowering time control. In addition, $l c 2$ mutant showed multiple phenotypes, including altered leaf angles, dark color, reversely rolled 

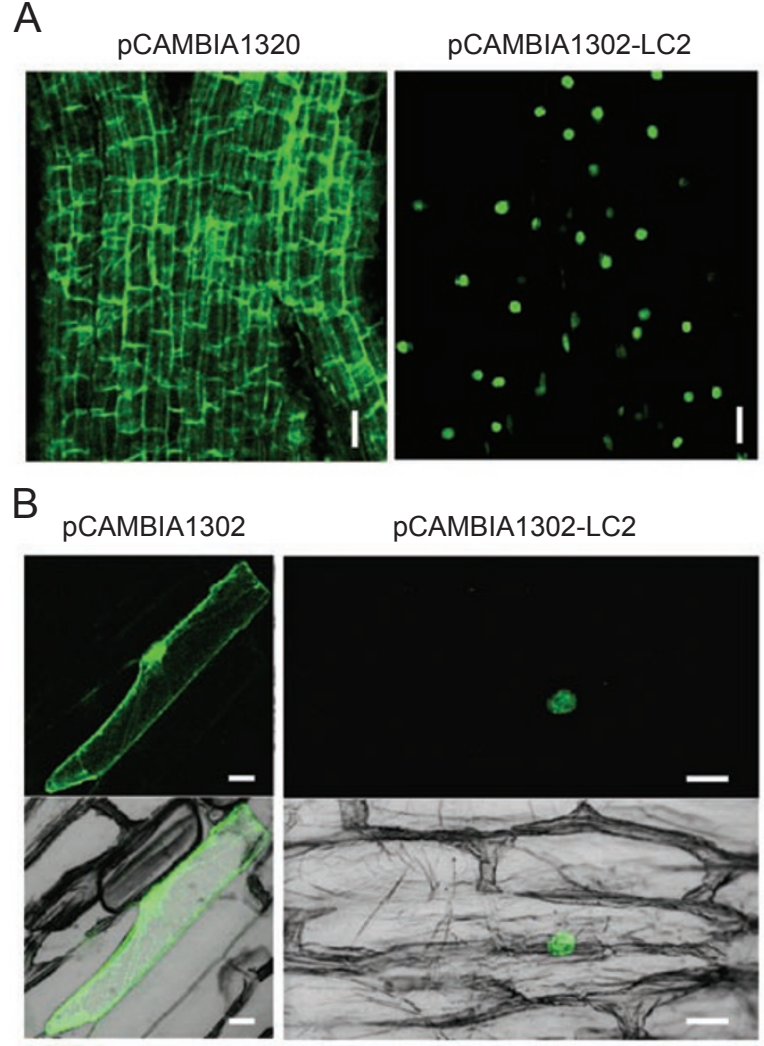

Figure 7 LC2 localizes to the nucleus. (A) The green fluorescence is ubiquitously distributed in the cytoplasm of transgenic rice plants harboring mock vector (pCAMBIA1302, left), whereas that in LC2-GFP harboring transgenic plants is accumulated in the nucleus (pCAMBIA1302-LC2, right). Bar $=20 \mu \mathrm{m}$. (B) The green fluorescence is ubiquitously distributed in the cytoplasm of onion epidermal cells harboring mock vector (pCAMBIA1302, left), whereas those in cells harboring LC2-GFP fusion proteins are accumulated to the nucleus (pCAMBIA1302-LC2, right). Bar $=20 \mu \mathrm{m}$. The onion epidermal cells were transiently transformed by gene particle bombardment, and observed after incubation for $24 \mathrm{~h}$.

leaves, late flowering and severe sterility, suggesting that LC2 is critical in rice architecture regulation, and VIN3like proteins may have different functions during plant development in addition to flowering time control, which is consistent with the differential expression pattern between LC2 and Arabidopsis VIN3 and wheat VIN3-like genes.

Our study results suggest a crucial role of LC2 in leaf inclination regulation, through modulating adaxial cell division of the collar. In rice, the lamina joint contributes significantly to leaf angle formation. Altered cell division or elongation in the adaxial surface of the collar will result in changed leaf angles. It has been shown the BRinduced rice lamina joint inclination was mainly due to the stimulated rapid expansion of collar adaxial cells [5]. The OsMDP1 (OsMADS47)-deficient seedlings had altered expansion of adaxial cells at leaf joint and showed enhanced lamina joint inclination under BR treatment [6], indicating the substantial effects of cell division or elongation at the lamina joint on leaf angle formation.

Leaves join onto the stem through leaf sheaths, however, no alteration was observed in the $l c 2$ sheath, suggesting the abnormal collar is the main reason for the altered leaf angle. In addition, increased adaxial cell division, but not cell elongation, in the collar of $l c 2$ further showed that LC2 serves as a negative regulator of cell division at the lamina joint to regulate leaf inclination. Although the numbers of leaf bulliform cells were increased under $L C 2$ deficiency, we propose that this mainly contributes to the leaf rolling phenotype (motor cells have an important role in leaf rolling [1, 44]), and the resultant changed physical shape and altered tissue flexibility might not lead to the bending of leaves.

The physiological effects of LC2 fit well with its expression pattern. During rice growth, the inclination of early leaves is much less than that of the later ones and consistently, $L C 2$ expression is much higher in early leaves (Figure 5B). In addition, $L C 2$ expression shows the opposite tendency along with the maturation of the later leaves (low expression at the early developmental stages of leaf to promote the cell division that results in the formation of the leaf angle, followed by an increase in its expression to inhibit cell division). The increased cell division in the $l c 2$ collar is consistent with the enhanced expression of genes promoting cell division. The increased cell division was also observed in the leaf bulliform cells and anther epidermal cells. LC2 is localized in the nucleus and may directly regulate the expression of multiple genes. The presence of the PHD finger motif, which is associated with chromatin-mediated transcriptional regulation, further indicates that LC2 may be involved in the relevant process through direct transcriptional regulation of corresponding genes. In addition, all three alleles of $l c 2$ mutant resulted in truncated LC2 proteins lacking a functional VID domain, which is conserved in VIN3-like proteins, suggesting the importance of the VID domain in LC2 function.

Previous studies have revealed the effects of plant phytohormones, especially BR, in regulating leaf angles. The expression of $L C 2$ is induced by BR, IAA and GA, and examination of transcripts of hormone-related genes suggested altered BR and GA signaling under $L C 2$ deficiency. As BR, IAA, and GA are involved in the regulation of leaf inclination, especially BR, it cannot be excluded that LC2 regulates leaf inclination by interacting with hormone-signaling pathways. However, although 
the expression of $L C 2$ is induced by $\mathrm{BR}, \mathrm{BR}$ signaling is possibly enhanced (indicated by reduced expression of CPD1) in lc2-1 mutant; and additionally, BR mainly functions to stimulate cell elongation, whereas $L C 2$ suppresses cell division. We would like to suggest that LC2 may regulate leaf inclination through a BR-independent pathway and may participate in the feedback control of BR signaling. Further studies on the interaction between $L C 2$ and BR and GA signaling will be needed to illustrate the detailed mechanisms.

Several rice MADS-box proteins including OsMADS22, OsMADS55, and OsMADS47 are involved in the regulation of leaf joint angles and BR responses $[6$, 23]. Considering that Arabidopsis VIN3 directly regulates the MADS-box proteins, it would be interesting to test whether there is a direct regulation of rice MADS genes by VIN3-like proteins.

As rice leaf angle is one of the important agronomic traits affecting the production, function study of $L C 2$ may contribute to plant architectural modifications through modulating the leaf inclination by molecular breeding.

\section{Materials and Methods}

\section{Plant materials and growth conditions}

Three alleles of the rice $l c 2$ mutants were identified from the japonica cultivar YunDao32 from spontaneous mutations. The $l c 2-$ 1 mutant was crossed with a rice indica variety MingHui63, and F1 plants were self-bred to produce the $F_{2}$ seeds for constructing the F2 mapping population. An allelic test was carried out through crossing and multiple generation analysis between the $l c 2-1$ and another 2 spontaneous mutations. The mutant seeds are available on request.

Rice plants were cultivated in the field at China National Rice Research Institute under the natural growing conditions. For growth of transgenic plants, seeds were germinated in sterilized water, and grown in a phytotron with a $12 \mathrm{~h}$ light $\left(28^{\circ} \mathrm{C}\right) / 12 \mathrm{~h}$ dark $\left(22^{\circ} \mathrm{C}\right)$ cycle. The angles between the leaf blades and the culms were measured with a protractor.

For qRT-PCR analysis, the collars were collected at 5 days after the complete elongation of the leaf blade and leaf sheath. The collars of last four leaves were collected at 0,5 , and 10 days after the complete elongation of leaf blade and leaf sheath.

For hormone treatments, 2-week-old seedlings were treated with $1 \mu \mathrm{M}$ 24-eBL (one of the most active BRs, E1641; Sigma), $10 \mu \mathrm{M}$ GA3 (gibberellic acid, G7645, Sigma) and $100 \mu \mathrm{M} \mathrm{ABA}$ (A1049; Sigma). The treated materials were collected at different time points and used for RNA extraction.

\section{Scanning electron microscopy}

The 1-cm-long lamina joint of the flag leaf was excised from 1c2 and WT plants 7 days after tassel, then fixed in FAA (formalin/ acetic acid $/ 50 \%$ ethanol, 2:1:17, v/v/v) overnight. After dehydration in a graded ethanol series, the samples were critical-point dried for $4 \mathrm{~h}$ (Hitachi critical point dryer, HCP-2; Hitachi Koki Co.
Ltd., Japan), sputter-coated with gold in an E-100 ion sputter (Mitocity, Japan), and observed with a scanning electron microscope (Hitachi S-450; Japan).

Cross section of leaf, cross and longitudinal sections of lamina joint, and measurement of cell layers and cell length

The lamina joints (from the same leaf of $1 \mathrm{c} 2$ and WT plants) and leaf blades (from the flag leaf of $1 \mathrm{c} 2$ and WT plants at same growth stage) were collected, fixed in FAA overnight, and dehydrated through a graded ethanol series. Samples were embedded in Epon812 resin (Fluka) and polymerized. The cross sections (3 $\mathrm{mm}$ ) of leaf or collar and longitudinal sections of lamina joint were cut and stained briefly with filtered $1 \%$ toluidine blue. Sections were microscopically examined and photographed to measure the cell layers and cell lengths. To avoid the inaccuracies from sampling, three middle vascular bundles at the adaxial surface were used for microscopic observation and measurement of cell layers and cell lengths.

\section{Mapping and cloning of $L C 2$}

LC2 was mapped primarily with SSR and STS markers, using 214 F2 mutant plants. The LC2 locus was further mapped within a $64 \mathrm{~kb}$ region between STS markers HS37 and HS52 on two overlapping BAC clones OSJNBa0050G13 and P0463E12 on chromosome 2, using 1741 F2 mutants. New molecular markers were then developed by comparing the original or cleaved amplified polymorphic sequence between indica var. 9311 and japonica var. Nipponbare according to the published data (http://www.ncbi. nlm.nih.gov). The developed PCR-based molecular markers in this study include RM7451 (5'-TAA TAC GAG CAG CGA TCG TG-3', 5'-GCT AAT TGC AGC TTG TGT CG-3'), HS11 (5'-TCG GTA ACG GTA GAG GAT TG-3', 5'-TGT GGA GTA TGT ATC CTT TCA G-3'), HS15 (5'-AAC CTA CCA CTG CCA TTG C-3', 5'-GGC ATT ATC CAT ACC AGC AG-3'), HS17 (5'-GCA CTA ATG GCT CAT CTC GT-3', 5'-CGA GAA AGG GAT TGT AGT GC-3'), HS18 (5'-CGA AAC ACA AGT GAA CGG T-3', 5'-GAT TTG CCT TGA TAC TAT TCT G-3'), HS27 (5'-GCA GCT TCC TGG TTC ACA C-3', 5'-GAA CAA TCA CAT AAT CGA GGG3'), HS37 (5'-ACA GAC AAG GGA AGG AAA CC-3', 5'-ACG AGC AAC AGG TGG TAG C-3'), HS38 (5'-GTT GCT CTT CAC ACC ACA GT-3', 5'-CCG ATT ATT ACG ATG GAA G-3'), HS45 (5'-ACT CTC CCC TCC TCT TCC A-3', 5'-AGG CTA GGA GTT AAC CTC GC-3'), and HS52 (5'-GCA CAT GGG AAG AAG TAA G-3', 5'-CCA ACA TCA GAA AGA AGA CAG-3').

To define the molecular lesions of $1 \mathrm{c} 2,12 \mathrm{~kb}$ genomic DNA of $1 \mathrm{c} 2$ and corresponding WT variety (YunDao32) were amplified by PCR. The PCR products were sequenced and a candidate gene was amplified from both 1c2 and Nipponbare genomic DNA using different primers: HSD1 (5'-TGG ATT ATG ATT TGT GAG GAA G-3' and 5'-AGG GTG AGG TGA GGC TTT T-3'), HSD2 (5'-GCT CTG AAC TAT GAA CAC GAA C-3' and 5'-TCA ACC CAA CAA ACC AAT CA-3'), HSD3 (5'-TGG TGA TGG CTC AGG TCT C-3' and 5'-ATC AGC CAA TCC TTA CCA TC-3'), HSD4 (5'-GGT AGC AGC AGC CTA TCC T-3' and 5'-AGT CGG ACA AGA AGC AAA CA-3'), HSD5 (5'-ACT CCA GTA AGA CAG TGT GCT A-3' and 5'-GCA GAA GCG AGC ATT ACT T-3'), HSD6 (5'-TGA CTG GCA AAT CAT CTG GT-3' and 5'GGA CCA ATG ATG ATG ACA CTA C-3'), HSD7 (5'-CTT GAT GGT GGT TAT TAC TGC-3' and 5'-CTG GCT TAG TAA CTT 
GGT TCA-3'), HSD8 (5'-AGT GTT GGA TTC TTC CTT CTC-3' and 5'-CCT CAG CCT CCA GTT TCT T-3'), HSD9 (5'-CCG AGC AAT ACT TGA CTG TTT-3' and 5'-GAC TGA CCA CCA CTG TTT GC-3'), HSD10 (5'-GCT CGT TCT CAT CAA GTC CA-3', 5'-AAG AGC CAT TTT GAA GGA TG-3'), HSD11 (5'AGC AGA ACC AGA GAA GCG A-3' and 5'-CTG CTA CGA AAG AAT GGG AC-3'), HSD12 (5'-GGC TGG AAT GTG AGG GTT A-3' and 5'-GCA AGG GTC TAA ACC GAA C-3'), HSD13 (5'-GAA ACA TTG GGA TGA AGC AG-3' and 5'-TCC TTC CTC TTC TTC GGT CT-3'). Obtained sequences were analyzed with DNAMAN (version 5.2.2) software.

\section{Complemented expression of LC2}

A 7.4-kb genomic DNA fragment containing the entire $L C 2$ coding region and $1.8 \mathrm{-kb}$ upstream sequences was isolated from BAC OSJNBb0003C06 and subcloned into the binary vector pCAMBIA1300 for complementation studies. The resultant plasmid was transformed into $l c 2$ mutant through Agrobacteriummediated transformation using immature embryos as materials [45]. Complementary expression of $L C 2$ was measured by qRT$\mathrm{PCR}$, and confirmed positive transgenic lines were used for further analysis.

\section{Semiquantitative RT-PCR analysis}

RT-PCR analysis was performed to examine the expression of $H 4$ and EXB6 at the lamina joints of flag leaf of WT, $l c 2-1$, or lc2-1 pLC2:LC2 plants. Total RNAs were extracted from lamina joints of various plants and used to synthesize the first-strand cDNA, which was then used as PCR templates. Equal amounts of cDNAs were used for PCR amplification using the primers as follows: $E X P B 6$ (5'-TGC AAG GGG CAT CCT GTG A-3' and 5'CGT TGC CGC AGG AGG TCA T-3'); $H 4$ (5'-ATC CGC GAC GCC GTC ACC T-3' and 5'-TCC CAT CGA AAC CCT AGC TCC T-3'). Rice ACTIN gene (Os03g50890) was amplified using primers (5'-GAA CTG GTA TGG TCA AGG CTG-3' and 5'-ACA CGG AGC TCG TTG TAG AAG-3'), and used as an internal positive control.

\section{$q R T-P C R$ analysis}

qRT-PCR analysis was performed to examine the expression pattern of $L C 2$ in various tissues and lamina joints of different rice leaves, to identify the transgenic $l c 2$ lines with complementary expressions of $L C 2$ and to detect the transcripts of cell division or cell elongation-related or BR-related genes among WT, $l c 2-1$, and lc2-1 pLC2:LC2 plants.

Total RNAs were extracted using Trizol solution (Invitrogen) and reverse-transcribed into first-strand cDNA according to the manufacturer's instructions (TaKaRa). Quantitative analyses were carried out on the Rotor-Gene real-time thermocycler R3000 (Corbett Research) with Real-Time PCR Master Mix (Toyobo). The primers used were as follows: LC2 (5'-AGC ATC AGC TTT GGA CGA GGA-3' and 5'-CAG TTG GTG GAA TAG AGC CAG AAT-3'), $R 2$ (5'-TCT GCA CCT CCA CTT CGC TCA-3' and 5'TAG GTG GTG GCC TTG GAA GCT-3'), H3 (5'-AGC GAA GAG GAG ATG GCC CGT-3' and 5'-AGG AGC TCC GTG CTC TTC TGG T-3'), CDKA; 1 (5'-ATC ACG GCA ACA TCG TCA GG-3' and 5'-AGT AAG CAA CGC CGC GGA GTA-3'), CDKA;2 (5'-ACC ACC GCA TAG TCA AAT CGT T-3' and 5'-ACC ACA ATG TCA CCA CCT CGT GA-3'), XTRl (5'-AGC CGT ACA
TCC TGC AGA CGA-3' and 5'-GCC CAG GTC CTT GCT GTT CT-3'), CPD1 (5'-TCT TCT CCA TCC CCT TTC CTC T-3' and 5'-TCA AGA AGC TCC TCA ACC ATG T-3' [44]), DWF4 (5'-AGT CGC GTG CTG CCA TTC T-3' and 5'-AGC TCA GCA AGA GGT CCA GGA T-3' [3]), BRI1 (5'-TAC CAG AGC TTC AGA TGC ACC A-3' and 5'-AGT AGC TCA GGG TCG AAG ACA T-3' [13]), OsGA2ox3 (5'-TTC TTC GTC AAC GTC GGC GAC TCG TTG C-3' and 5'-TCT CAA ACT GGG CCA GCC TGT TGT CTC C-3' [39]), OsGA20ox2 (5'-TAC TAC AGG GAG TTC TTC GCG GAC AGC A-3' and 5'-TGT GCA GGC AGC TCT TAT ACC TCC CGT T-3' [39]), OsIAA1 (5'-GCC GCT CAA TGA GGC ATT-3' and 5'-GCT TCC ACT TTC TTT CAA TCC AA-3' [40]), OsIAA9 (5'-AAG AAA ATG GCC AAT GAT GAT CA-3' and 5'- CCC ATC ACC ATC CTC GTA GGT-3' [40]), and OsIAA24 (5'-GGC TTG TGC TCT TCG TTG CT-3' and 5'-CCT CTT GGA TTC AGA AAC ACT GAA-3' [40]).

The transcript levels of the examined genes were normalized with those of ACTIN and the relative expressions were compared with that of WT plants. All the experiments were repeated for three times, and the data were statistically analyzed and presented as means plus standard error (SE).

\section{Promoter-reporter gene fusion studies}

A $1.8-\mathrm{kb}$ promoter region of LC2 gene that was used in the complementation analysis to drive the expression of LC2 was cut from BAC OsJNBb0003C06 by restriction enzymes BamHI and Pst I, and then subcloned into the pCAMBIA1300+pBI101 vector [46]. The resultant promoter GUS construct was transformed into rice genome by Agrobacterium-mediated transformation and the selected positive transgenic plants were used for detecting the GUS activities according to Jefferson et al. [47].

\section{Subcellular localization of LC2}

The whole LC2 coding region was amplified with primes LC2-5 (5'-GAAGATCTA ATG GAT CCA CCC TAC GCA G-3', added $B g l$ II site underlined) and $L C 2-6$ (5'-GGACTAGTA TGC CAA AGT TCC ATG CAG A-3', added SpeI site underlined), and subcloned into the binary vector pCAMBIA1302, resulting in an $\mathrm{N}$-terminal fusion to GFP. The resultant vector was sequenced to confirm the in-frame fusion of $L C 2$ and GFP, and used for rice transformation. Root apices from 5-day-old transgenic plants harboring p35S:LC2-GFP (pCAMBIA1302-LC2) were observed through a confocal laser scanning microscopy (Bio-Rad Lasersharp 2000; Hercules, CA, USA) with a Kr/Ar laser 488 (FITC488, Zeiss LSM500; Herts, UK), and those of transgenic plants harboring vector pCAMBIA1302 were observed as control.

Transient transformation of onion epidermal cells was performed through gene particle bombardment (empty vector pCAMBIA1302 was used as control) [48]. Transformed onion cells were incubated for $24 \mathrm{~h}$ and then observed with a confocal laser scanning microscope (Zeiss LSM 510 META).

\section{Acknowledgments}

The study was supported by the state key project of basic research (2005CB120803), High-tech program (2006AA10A102, 2006AA10Z160), Chinese Academy of Sciences (KSCX2-YWN-016) and the National Science Foundation of China (30670187, 30870145). We thank Ms Shu-Ping Xu for help on rice transfor- 
mation and Mr Xiao-Yan Gao for sections and SEM observations.

\section{References}

1 Hoshikawa K. The growing rice plant: An anatomical monograph. 1st Edition, Okyo: Nosan Gyoson Bunka Kyokai (Nobunkyo), 1989.

2 Sinclair TR, Sheehy JE. Erect leaves and photosynthesis in rice. Science 1999; 283:1455.

3 Sakamoto T, Morinaka Y, Ohnishi T, et al. Erect leaves caused by brassinosteroid deficiency increase biomass production and grain yield in rice. Nat Biotechnol 2006; 24:105-109.

4 Hong Z, Ueguchi-Tanaka M, Matsuoka M. Brassinosteroids and rice architecture. J Pestic Sci 2004; 29:184-188.

5 Cao HP, Chen SK. Brassinosteroid-induced rice lamina joint inclination and its relation to indole-3-acetic acid and ethylene. J Plant Growth Regul 1995; 16:189-196.

6 Duan K, Li L, Hu P, Xu ZH, Xue HW. A brassinolidesuppressed rice MADS-box transcription factor, OsMDP1, has a negative regulatory role in BR signaling. Plant $J$ 2006; 47:519-531.

7 Li ZK, Paterson AH, Pinson SRM, Khush GS. A major gene, Tal and QTLs affecting tiller and leaf angles in rice. Rice Genetics Newslett 1998; 15:154-156.

8 Li ZK, Paterson AH, Pinson SRM, Stansel JW. RFLP facilitated analysis of tiller and leaf angles in rice (Oryza sativa $\mathrm{L}$ ). Euphytica 1999; 9:79-84.

9 Hong Z, Ueguchi-Tanaka M, Shimizu-Sato S, et al. Loss-offunction of a rice brassinosteroid biosynthetic enzyme, C-6 oxidase, prevents the organized arrangement and polar elongation of cells in the leaves and stem. Plant J 2002; 32:495508.

10 Hong Z, Ueguchi-Tanaka M, Umemura $\mathrm{K}$, et al. A rice brassinosteroid-deficient mutant, ebisu dwarf (d2), is caused by a loss of function of a new member of cytochrome P450. Plant Cell 2003; 15:2900-2910.

11 Wada K, Marumo S, Ikekawa N, Morisaki M, Mori K. Brassinolide and homobrassinolide promotion of lamina inclination of rice seedlings. Plant Cell Physiol 1981; 22:323-325.

12 Wada K, Marumo S, Abe $\mathrm{H}$, et al. A rice lamina inclination test - a micro-quantitative bioassay for brassinosteroids. $\mathrm{Ag}$ ric Biol Chem 1984; 48:719-726.

13 Yamamuro C, Ihara Y, Wu X. Loss of function of a rice brassinosteroid insensitivel homolog prevents internode elongation and bending of the lamina joint. Plant Cell 2000; 12:1591-1605.

14 Morinaka Y, Sakamoto T, Inukai Y, et al. Morphological alteration caused by brassinosteroid insensitivity increases the biomass and grain production of rice. Plant Physiol 2006; 141:924-931.

15 Bai MY, Zhang LY, Gampala SS, et al. Functions of OsBZR1 and 14-3-3 proteins in brassinosteroid signaling in rice. Proc Natl Acad Sci USA 2007; 104:13839-13844.

16 Wu CY, Trieu A, Radhakrishnan P, et al. Brassinosteroids regulate grain filling in rice. Plant Cell 2008; 20:2130-2145.

17 Nakamura A, Fujioka S, Takatsuto S, et al. Involvement of C-22-hydroxylated brassinosteroids in auxin-induced lamina joint bending in rice. Plant Cell Physiol 2009; 50:1627-1635.

18 Shimada A, Ueguchi-Tanaka M, Sakamoto T, et al. The rice
SPINDLY gene functions as a negative regulator of gibberellin signaling by controlling the suppressive function of the DELLA protein, SLR1, and modulating brassinosteroid synthesis. Plant J 2006; 48:390-402.

19 Komatsu K, Maekawa M, Ujiie S, et al. LAX and SPA: major regulators of shoot branching in rice. Proc Natl Acad Sci USA 2003; 100:11765-11770.

20 Tanaka A, Nakagawa H, Tomita C, et al. BRASSINOSTEROID UPREGULATED 1, encoding a helix-loop-helix protein, is a novel gene involved in brassinosteroid signaling and controls bending of the lamina joint in rice. Plant Physiol 2009; 151:669-680.

21 Lee J, Park JJ, Kim SL, Yim J, An G. Mutations in the rice liguleless gene result in a complete loss of the auricle, ligule, and laminar joint. Plant Mol Biol 2007; 65:487-499.

22 Wang DK, Zhang HX, Hu GC, Fu YP, Si HM, Sun ZX. Genetic analysis and identification of a large leaf angles (lla) mutant in rice. Chinese Sci Bull 2005; 50:492-494.

23 Lee S, Choi SC, An G. Rice SVP-group MADS-box proteins, OsMADS22 and OsMADS55, are negative regulators of brassinosteroid responses. Plant J 2008; 54:93-105.

24 Wang L, Xu YY, Zhang C, et al. OsLIC, a novel CCCH-type zinc finger protein with transcription activation, mediates rice architecture via brassinosteroids signaling. PLOS ONE 2008; 3:e3521. DOI: 10.1371/journal.pone.0003521.

25 Zhang LY, Bai MY, Wu J, et al. Antagonistic HLH/bHLH transcription factors mediate brassinosteroid regulation of cell elongation and plant development in rice and Arabidopsis. Plant Cell 2009; 21:3767-3780.

26 Shi Z, Wang J, Wan X, Shen G, Wang X, Zhang J. Overexpression of rice $\mathrm{OsAGO7}$ gene induces upward curling of the leaf blade that enhanced erect-leaf habit. Planta 2007; 226:99-108.

27 Sung S, Amasino RM. Vernalization in Arabidopsis thaliana is mediated by the PHD finger protein VIN3. Nature 2004; 427:159-164.

28 Sung S, Schmitz RJ, Amasino RM. A PHD finger protein involved in both the vernalization and photoperiod pathways in Arabidopsis. Genes Dev 2006; 20:3244-3248.

29 Fu D, Dunbar M, Dubcovsky J. Wheat VIN3-like PHD finger genes are up-regulated by vernalization. Mol Genet Genomics 2007; 277:301-313.

30 Aasland R, Gibson TJ, Stewart AF. The PHD-finger: implications for chromatin-mediated transcriptional regulation. Trends Biochem Sci 1995; 20:56-59.

31 Mariann B. The PHD finger, a nuclear protein-interaction domain. Trends Biochem Sci 2006; 31:35-40.

32 Aspberg A, Miura R, Bourdoulous S, et al. The C-type lectin domains of lecticans, a family of aggregating chondroitin sulfate proteoglycans, bind tenascin-R by protein-protein interactions independent of carbohydrate moiety. Proc Natl Acad Sci USA 1997; 94:10116-10121.

33 Pankov R, Yamada KM. Fibronectin at a glance. J Cell Sci 2002; 115:3861-3863.

34 Fabian-Marwedel T, Umeda M, Sauter M. The rice cyclindependent kinase-activating kinase R2 regulates S-phase progression. Plant Cell 2002; 14:197-210.

35 Renaudin JP, Doonan JH, Freeman D, et al. Plant cyclins: a unified nomenclature for plant A-, B- and D-type cyclins 
based on sequence organisation. Plant Mol Biol 1996; 32:1003-1018.

36 Sauter M. Differential expression of a CAK (cdc2-activating kinase)-like protein kinase, cyclins and $c d c 2$ genes from rice during the cell cycle and in response to gibberellin. Plant $J$ 1997; 11:181-190.

37 Lee Y, Kende H. Expression of $\beta$-expansins is correlated with internodal elongation in deepwater rice. Plant Physiol 2001; 127:645-654.

38 Uozu S, Tanaka-Ueguchi M, Kitano H, Hattori K, Matsuoka M. Characterization of XET-related genes of rice. Plant Physiol 2000; 122:853-859.

39 Sakamoto T, Miura K, Itoh H, et al. An overview of gibberellin metabolism enzyme genes and their related mutants in rice. Plant Physiol 2004; 134:1642-1653.

40 Jain M, Kaur N, Garg R, Thakur JK, Tyagi AK, Khurana JP. Structure and expression analysis of early auxin-responsive Aux/IAA gene family in rice (Oryza sativa). Funct Integr Genomics 2006; 6:47-59.

41 Bienz M. The PHD finger, a nuclear protein-interaction domain. Trends Biochem Sci 2006; 31:35-40.

42 Wood CC, Robertson M, Tanner G, Peacock WJ, Dennis ES, Helliwell CA. The Arabidopsis thaliana vernalization re- sponse requires a polycomb-like protein complex that also includes VERNALIZATION INSENSITIVE 3. Proc Natl Acad Sci USA 2006; 103:14631-14636.

43 Sheldon CC, Finnegan EJ, Dennis ES, Peacock WJ. Quantitative effects of vernalization on FLC and SOC1 expression. Plant J 2006; 45:871-883.

44 Mori M, Nomura T, Ooka $\mathrm{H}$, et al. Isolation and characterization of a rice dwarf mutant with a defect in brassinosteroid biosynthesis. Plant Physiol 2002; 130:1152-1161.

45 Hiei Y, Ohta S, Komari T, Kumashiro T. Efficient transformation of rice (Oryza sativa L.) mediated by Agrobacterium and sequence analysis of the boundaries of the T-DNA. Plant $J$ 1994; 6:271-282.

46 Liu W, Xu ZH, Luo D, Xue HW. Roles of OsCKI1, a rice casein kinase I, in root development and plant hormone sensitivity. Plant J 2003; 36:189-202.

47 Jefferson RA, Kavanagh TA, Bevan MW. GUS fusions: betaglucuronidase as a sensitive and versatile gene fusion marker in higher plants. EMBO J 1987; 6:3901-3907.

48 Lou Y, Gou JY, Xue HW. PIP5K9, an Arabidopsis phosphatidylinositol monophosphate kinase, interacts with a cytosolic invertase to negatively regulate sugar-mediated root growth. Plant Cell 2007; 19:163-181. 